\section{Effectiveness of animal care and use program performance measurements}

\section{To the editor:}

Performance measurement has been well recognized as an important tool for improving the quality of health care ${ }^{1}$. However, whether it can also improve the quality of animal care and use programs is not clear. We analyzed performance metric data of the Department of Veterans Affairs research facility's animal care and use programs collected annually from 2011, the first year of our data collection, through 2016. The Department of Veterans Affairs health care system is the largest integrated health care system in the US with more than 70 facilities conducting research involving laboratory animals each year. As part of the quality assurance program, facilities were required to conduct regulatory audits of all active animal research protocols once every three years ${ }^{2,3}$. As shown in Table 1, among a total of 14 animal care and use program performance metrics, nine showed improvements, ranging from $56 \%$ to $100 \%$, five remained unchanged, and none deteriorated during the study period. The five performance metrics that showed no improvement from 2011 through 2016 were those with very small initial noncompliant rates, namely, less than $0.3 \%$ in 2011 . On the other hand, those that showed improvement were those with initial noncompliant rates of $0.89 \%$ to $5.57 \%$. The majority of the improvement in these nine performance metrics occurred within the first two to three years of the study period. We conclude that performance measurement is an effective tool in improving the performance of animal care and use programs. The issue challenging us today is not whether we should continue to measure performance, but how to improve current performance measurements to maximize benefits. Our current performance metrics undoubtedly have not covered the full scope of the animal care and use program. In view of the encouraging results reported here, we plan to expand our animal care and use program performance measurements by adding additional metrics in the future.

\section{Min-Fu Tsan ${ }^{1} \&$ Yen Nguyen ${ }^{2}$}

${ }^{1}$ McGuire Research Institute, Richmond, VA. ${ }^{2}$ Office of Research Oversight, Department of Veterans Affairs, Washington, DC. Correspondence should be addressed to M.T. (minfu.tsan@gmail.com).

1. Cassel, C.K., Conway, P.H., Delbanco, S.F., Jha, A.K., Saunders, R.S., Lee, T.H. N. Engl. J. Med. 371, 2145-2147 (2014).

2. Tsan, M.F., Bannerman, D.D., Lakshman, R., McVicker, B., Gao, B., Nguyen, Y., et al. Fed. Prac. 32, 58-63 (2015).

3. Tsan, M.F., Grabenbauer, M., Nguyen, Y. PLoS ONE 11, e0162141. doi:10.1371/journal.pone.0162141 (2016).

4. Agresti A. Analysis of Ordinal Categorical Data (John Wiley \& Sons, New York, 1984).

TABLE 1 | VA animal care and use program performance measurements

\begin{tabular}{|c|c|c|c|c|c|c|c|c|}
\hline & 2011 & 2012 & 2013 & 2014 & 2015 & 2016 & $P$ value $^{1}$ & $\begin{array}{l}\text { Change } \\
(\%)^{2}\end{array}$ \\
\hline Initiated before IACUC approval & $2(0.15 \%)^{4}$ & $1(0.08 \%)$ & $2(0.17 \%)$ & $1(0.09 \%)$ & $0(0.00 \%)$ & $1(0.11 \%)$ & 0.5118 & $\mathrm{~N} / \mathrm{A}$ \\
\hline Completed without IACUC approval & $0(0.00 \%)$ & $1(0.08 \%)$ & $0(0.00 \%)$ & $0(0.00 \%)$ & $0(0.00 \%)$ & $0(0.00 \%)$ & 0.4682 & $\mathrm{~N} / \mathrm{A}$ \\
\hline Initiated before $R \& D C$ approval & $1(0.07 \%)$ & $2(0.16 \%)$ & $4(0.35 \%)$ & $2(0.19 \%)$ & $2(0.20 \%)$ & $16(1.71 \%)$ & 2.0000 & $\mathrm{~N} / \mathrm{A}$ \\
\hline Continued research during lapse & -5 & $25(1.94 \%)$ & $6(0.52 \%)$ & $12(1.12 \%)$ & $2(0.20 \%)$ & $3(0.32 \%)$ & 0.0000 & 84 \\
\hline \multicolumn{9}{|l|}{ For-cause suspension/termination } \\
\hline Number of active protocols & - & - & 3,203 & 3,119 & 3,087 & 2,837 & & \\
\hline Number of protocols suspended & - & - & $41(1.28 \%)$ & $30(0.96 \%)$ & $22(0.71 \%)$ & $11(0.39 \%)$ & 0.0001 & 70 \\
\hline Due to animal welfare concerns & - & - & $7(0.22 \%)$ & $11(0.35 \%)$ & $7(0.23 \%)$ & $6(0.21 \%)$ & 0.7455 & N/A \\
\hline Without scope of practice & $170(3.45 \%)$ & $276(5.99 \%)$ & $61(1.20 \%)$ & $23(0.48 \%)$ & $59(1.27 \%)$ & $13(0.32 \%)$ & 0.0000 & 91 \\
\hline Working outside of scope of practice & $1(0.02 \%)$ & $1(0.02 \%)$ & $1(0.02 \%)$ & $0(0.00 \%)$ & $0(0.00 \%)$ & $0(0.00 \%)$ & 0.1403 & $\mathrm{~N} / \mathrm{A}$ \\
\hline Lapse in required training & $276(5.60 \%)$ & $186(4.04 \%)$ & $119(2.35 \%)$ & $138(2.85 \%)$ & $175(3.77 \%)$ & $55(1.35 \%)$ & 0.0000 & 76 \\
\hline Without initial training & $93(1.89 \%)$ & $26(0.56 \%)$ & $19(0.38 \%)$ & $24(0.50 \%)$ & $16(0.34 \%)$ & $14(0.34 \%)$ & 0.0000 & 82 \\
\hline Lapse in annual continuing training & $183(3.71 \%)$ & $160(3.48 \%)$ & $100(1.97 \%)$ & $114(2.36 \%)$ & $159(3.43 \%)$ & $41(1.01 \%)$ & 0.0000 & 73 \\
\hline
\end{tabular}

